\title{
PCSK9 and lipid metabolism and atherosclerosis: animal models
}

\author{
Godfrey S. Getz', Catherine A. Reardon ${ }^{2}$ \\ 'Department of Pathology, University of Chicago, Chicago, IL 60637, USA. \\ ${ }^{2}$ Ben May Institute, University of Chicago, Chicago, IL 60637, USA.
}

Correspondence to: Dr. Godfrey S. Getz, Department of Pathology, Department of Pathology, University of Chicago, 5841 South Maryland Avenue, MC 1089, Chicago, IL 60637, USA. E-mail: getz@bsd.uchicago.edu

How to cite this article: Getz GS, Reardon CA. PCSK9 and lipid metabolism and atherosclerosis: animal models. Vessel Plus 2021;5:17. https://dx.doi.org/10.20517/2574-1209.2020.70

Received: 12 Nov 2020 First Decision: 17 Dec 2020 Revised: 21 Dec 2020 Accepted: 23 Dec 2020 Published: 17 Apr 2021

Academic Editor: Ping-Yen Liu Copy Editor: Xi-Jun Chen Production Editor: Xi-Jun Chen

\begin{abstract}
PCSK9 is a secreted protein that binds to the LDL receptor on the cell surface, is endocytosed along with the receptor, and interferes with its ability to recycle to the cell surface. Instead the receptor is directed to the lysosome where it is degraded, leading to decreased levels of the receptor on the cell surface and decreased internalization of LDL. In this review, we will discuss how mice deficient in the Pcsk9 gene or hepatic overexpression due to transgenesis or viral vector mediated expression contributed to our understanding of the in vivo role of PCSK9. In addition, we will discuss how the discovery of PCSK9 and its gain of function mutants has greatly facilitated the manipulation of animals modelling atherosclerosis to induce hypercholesterolemia and atherosclerosis.
\end{abstract}

Keywords: PCSK9, LDL receptor, hyperlipidemia, atherosclerosis, mice, pig, animal models of atherosclerosis, knockout, transgenic, viral mediated

\section{INTRODUCTION}

Much of the concern with the approach to atherosclerotic cardiovascular disease hinges on the role of the LDL receptor (LDLR), stemming from the seminal work of Drs. Goldstein and Brown ${ }^{[1]}$. Two children with homozygous familial hypercholesterolemia, the most common form of autosomal dominant hypercholesterolemia, stimulated their interest in understanding the genetic basis for their hypercholesterolemia. Such subjects are at risk of early death from atherosclerotic cardiovascular disease due to mutations that affect the level or function of the LDLR. Other forms of autosomal dominant 
hypercholesterolemia are related to increased ApoB100 production and secretion and gain of function mutants of proprotein convertase subtilisin/kexin type 9 (PCSK9). For the most part, the function of PCSK9 with respect to hypercholesterolemia depends on its interaction with the LDLR. This will be the thrust of this review.

\section{PCSK9 AND LIPID METABOLISM AND ATHEROSCLEROSIS}

Pathways regulating cell surface levels of the LDLR

In order to understand the function of PCSK9, it is necessary to summarize the cellular trajectory of the LDLR. The receptor is present on cell membranes in clathrin coated pits where it interacts with extracellular LDL. The receptor-LDL complex is endocytosed and upon acidification of the endosome, the LDL is released from the receptor and is degraded in the lysosome liberating its cholesterol. The LDL derived cholesterol is used for membrane assembly, used for lipoprotein assembly, or esterified by acyl cholesteryl acyl transferase for storage. The LDLR then recycles to the cell surface for repeated cycles of LDL endocytosis. On average, each receptor recycles about 20 times. Cholesterol in the endoplasmic reticulum (ER) plays an important regulatory role by interacting with a series of membrane bound ER proteins that transport the precursors of the sterol responsive element binding protein transcription factors (SREBP1 and SREPB2) to the Golgi when cellular cholesterol levels are low or depleted. When cellular cholesterol is high, the binding proteins and the SREBP precursors are retained in the ER. The processing of the SREBP precursors in the Golgi involves 2 proteases that release the active transcription factor for transport to the nucleus. One of these proteases, site 1 protease, is a member of the subtilisin family (number 8), to which PCSK9 belongs. Among the genes induced under low cholesterol conditions are those encoding HMG-CoA reductase (the rate limiting enzyme for cholesterol biosynthesis) and the LDLR (involved in the acquisition of cholesterol packaged in the LDL particle). The rationale for the widespread use of the statins in the treatment of hypercholesterolemia relates to their inhibition of HMG-CoA reductase and hence cholesterol biosynthesis.

This very brief description indicates that cellular cholesterol homeostasis is maintained by regulated transcriptional feedback control of both cellular cholesterol biosynthesis and the acquisition of extracellular cholesterol packaged in LDL. The latter process, especially in the liver, is the most important pathway regulating plasma LDL levels. However, LDLR is expressed in most of the cells and tissues of the body. Indeed, most of the early experiments performed by Brown and Goldstein were with cultured human fibroblasts or the adrenal gland from which the receptor was first purified.

Though the transcriptional regulation of the LDLR gene is critical for the control of plasma cholesterol levels, there are also post-transcriptional mechanisms that modulate the level of the receptor on the cell surface. One of these involves the ubiquitination of the cytoplasmic tail of the receptor by the E3 ligase inducible degrader of the LDL receptor (IDOL) leading to proteasomal degradation of the receptor ${ }^{[2]}$. IDOL expression is positively regulated by the oxysterol sensing liver $\mathrm{X}$ receptor transcription factor. This will not be further discussed in this review. The second mechanism involves increased lysosomal degradation of the receptor mediated by PCSK9. The importance of PCSK9 in LDL cholesterol metabolism was discovered when mutations in the PCSK9 gene were identified in 2 French families with autosomal dominant hypercholesterolemia that were not linked to mutations in the LDLR and APOB genes ${ }^{[3]}$. There was an AT substitution at nucleotide 625 resulting in a serine to arginine change at amino acid residue 127 (p.S127R) in 1 family and a TC substitution at nucleotide 890 resulting in the amino acid alteration of p.F216L in the second family. Additional mutants have also been identified including the p.D374Y mutation ${ }^{[4,5]}$. The identification of PCSK9 mutations associated with hypercholesterolemia represented an important new chapter in the regulation of plasma LDL levels. 
It should be noted that the PCSK9 gene was originally determined to be identical to the neuronal apoptosis factor NARC-1 gene. While PCSK9 (NARC-1) is highly expressed in the liver where it plays an important role in regulating plasma cholesterol levels, the gene is ubiquitously expressed. The protein is known to regulate neuronal survival in the central nervous system, may regulate renal physiology in the kidney, and properties of cells in the vascular wall among other functions that are not related to regulating plasma LDL levels ${ }^{[6,7]}$.

\section{The structure and properties of PCSK9}

Human PCSK9 is a $72 \mathrm{kDa}$ secreted protein, containing 692 amino acids. It has 3 domains in addition to the 30 amino acid signal peptide: the prodomain (residues 31-152), the catalytic domain (residue 153-425), and a C terminal cysteine and histidine rich domain (residues 426-692) ${ }^{[8]}$. The original mutations described by Abifadel et al. ${ }^{[3]}$ are in the prodomain and catalytic domain, respectively. Pro-PCSK9 facilitates the transport of the LDLR from the ER to the Golgi where the latter is matured by glycosylation. Pro-PCSK9 is autocatalytically cleaved in the Golgi at residue Q152, though the prodomain (13kDa) remains noncovalently attached to the rest of the protein. This blocks its catalytic activity. In addition, autocatalytic cleavage is important for the secretion of PCSK9. A naturally occurring p.Q152H mutation prevents the autocatalytic cleavage and protein secretion. This mutation is associated with a $48 \%$ reduction of plasma LDL cholesterol (LDL-C) levels in humans ${ }^{[9]}$. In the Golgi, PCSK9 associates with sortilin which facilitates its secretion from the cell ${ }^{[10]}$.

PCSK9 heterodimer (62 $\mathrm{kDa}$ plus $13 \mathrm{kDa}$ prodomain) circulates in the plasma in free form or bound to LDL. In the plasma, there is 1 molecule of PCSK9 for each 500-1000 LDL particles. It also binds lipoprotein $(\mathrm{a})^{[11]}$, an independent risk factor for cardiovascular disease. However, it has little affinity for VLDL, chylomicrons, or remnants of chylomicrons.

\section{PCSK9 disrupts LDLR recycling}

PCSK9 regulates the number of LDLR molecules on the cell surface. The catalytic domain of PCSK9 binds to the extracellular epidermal growth factor-like repeat A domain of the LDLR on the cell surface and is endocytosed along with the receptor. In the endosome, the acidic $\mathrm{pH}$ leads to an interaction between the Cterminal domain of PCSK9 and the ligand binding domain of the LDLR leading to increased affinity between the molecules and preventing the LDLR from assuming the conformation needed for it to recycle to the plasma membrane. As a result, instead of recycling to the plasma membrane, the LDLR is directed to the lysosome where it is degraded, leading to decreased levels of cell surface LDLR and decreased internalization of LDL. PCSK9 mediated degradation of the LDLR is independent of its catalytic activity since similar reduction of hepatic LDLR was observed by the infusion of both wild type human PCSK9 (hPCSK9) and a catalytically inactive hPCSK9 mutant (p.S386A) into Pcsk9 knockout (Pcsk9-/-) mice e $^{[12]}$. Antibodies to the C-terminal domain of PCSK9 prevents the internalization of the PCSK9-LDLR complex ${ }^{[13]}$.

The majority of LDLR degradation occurs via the extracellular interaction of PCSK9 with the receptor. However, some degradation occurs in the secretory pathway in hepatocytes. It has been shown that a large percentage of ApoB, the major apoprotein on LDL and its precursor VLDL, is degraded within hepatocytes prior to secretion ${ }^{[14]}$. This occurs before ApoB is fully lipidated in the ER and is dependent on the LDLR ${ }^{[15]}$. PCSK9 expression has an impact on plasma triglycerides as well. The mechanism is not entirely clear, but it may be related to the decrease in cell surface LDLR leading to reduced degradation of ApoB in the presecretory pathway and to reduced uptake of nascent VLDL particles secreted by hepatocytes ${ }^{[1,117]}$. 
PCSK9 regulates LDL-C levels in humans. Polymorphisms in the PCSK9 gene generate either gain of function or loss of function resulting in hypercholesterolemia or hypocholesterolemia respectively ${ }^{[18,19]}$. Inhibition of the action of plasma PCSK9 by treatment with monoclonal antibodies that block its ability to bind to the LDLR reduces LDL-C levels ${ }^{[2,21]}$. Lipoprotein(a) levels are also reduced by PCSK9 monoclonal antibody treatment ${ }^{[22]}$. The effect on lipoprotein(a) may involve both LDLR-dependent and independent pathways. A newer approach uses antisense oligonucleotides to reduce hepatic PCSK9 synthesis by inhibiting translation results in sustained reductions in LDL-C $\mathrm{C}^{[20,23]}$.

\section{Regulation of LDLR and PCSK9 genes}

The regulation of the LDLR and PCSK9 genes can be complex depending on the setting. They are both regulated by cellular cholesterol homeostasis as they contain sterol regulatory elements in their promoters to which SREBP2 binds ${ }^{[24]}$. However, when stimulated by adiponectin receptor agonists, they are both increased in wild type mice, though the mechanisms differ ${ }^{[25]}$. In agonist treated human hepatoma HepG2 cells, PCSK9 transcription is activated through a PPAR dependent pathway while LDLR transcription involves SREBP2.

\section{Impact of PCSK9 on lipid metabolism in mouse models}

Mice have been useful to understand how PCSK9 influences lipid metabolism and atherosclerosis. Mice with global deficiency of the Pcsk9 gene (Pcsk9-/-) have a 2-3-fold increase in hepatic LDLR protein and very low plasma levels of LDL-C ${ }^{[2,22]}$. In contrast, transgenic expression of human PCSK9 in the liver using the ApoE promoter with liver specific enhancer results in an increment in plasma LDL-C levels close to levels in global $L d l r-/-$ mice $^{[28]}$. Additionally, LDLR protein in the liver was essentially eliminated ${ }^{[28]}$. Similar increase in LDL-C was observed in transgenic mice expressing human PCSK9 under the control of its endogenous promoter ${ }^{[29]}$. Recombinant adenoviral vectors have also been used to increase hepatic expression of murine ${ }^{[30]}$ and human ${ }^{[31,32]}$ PCSK9. Following injection of the recombinant virus into wild type mice, PCSK9 protein levels were increased in the liver in a dose-dependent manner and hepatic LDLR levels were reduced. The mice became hypercholesterolemic with a lipoprotein profile similar to that seen in chow fed $L d l r-/$ - mice. These effects on LDL-C levels are dependent on the presence of the LDLR since neither decreased nor increased expression of Pcsk9 in Ldlr-/- mice had an effect on LDL-C levels ${ }^{[30,33,34]}$.

PCSK9 is widely expressed in many tissues ${ }^{[27,35]}$. The tissue with the highest level of expression in mice is the liver. It is also highly expressed in the intestine with lower levels of expression observed in the kidney, spleen and aorta. Essentially all plasma PCSK9 is derived from the liver. In mice with liver specific deletion of Pcsk9, plasma levels of PCSK9 are undetectable. Despite the absence of plasma PCSK9, plasma LDL-C levels were only reduced by $60 \%$, suggesting a role of extrahepatic PCSK9 in regulating LDL-C levels ${ }^{[16,27]}$. PCSK9 is widely expressed in the intestine, and the small intestine is responsible for the assembly of triglyceride-rich lipoproteins containing dietary lipids. Extracellular recombinant PCSK9 is taken up by polarized human epithelial CaCo-2 cells resulting in stimulation of the synthesis and secretion of ApoB100 and $\mathrm{ApoB} 48$ due to increased $\mathrm{ApoB}$ mRNA and increased $\mathrm{ApoB}$ protein stability ${ }^{[3,37]}$. Increased ApoB stability is likely due to PCSK9 mediated increase in microsomal triglyceride transfer protein (MTP) and activity which facilitate the lipidation of ApoB leading to decreased intracellular ApoB degradation. Lipogenic gene expression was also increased by PCSK9. In vivo studies with wild type mice, Pcsk9-/- mice and mice transgenic for hPCSK9 reinforced the observations related to MTP expression in the small intestine made with the CaCo-2 cells. The stimulation of MTP occurred independent of LDLR. PCSK9 also enhanced Niemann-Pick C1-like (NPC1L1) levels and cholesterol uptake in CaCo-2 cells suggesting that PCSK9 might increase the absorption of cholesterol from the intestinal lumen into enterocytes for assembly into lipoproteins. LDLR degradation under the influence of PCSK9 is seen in lymphocytes which like the hepatocytes requires the autosomal recessive hypercholesterolemia adaptor protein that binds to the 
receptor's cytoplasmic tail ${ }^{[38,39]}$.

The ligands on lipoproteins recognized by the LDLR are ApoB100 and ApoE. As a subfraction of HDL contains ApoE, the level of HDL-C may be influenced by PCSK9. Indeed, the concentration of HDL, particularly the ApoE-containing HDL subfraction, is reduced in mice deficient in Pcsk ${ }^{[26,40]}$. PCSK9 binds other lipoprotein receptors, such as the VLDL receptor, ApoE receptor 2, and LRP-1, but how this impacts processes mediated by these receptors is less well understood.

\section{Impact of PCSK9 on atherosclerosis in mouse models}

In almost all animal models, the presence of hypercholesterolemia due to increased levels of ApoBcontaining lipoproteins is necessary to drive atherogenesis. The major lipoprotein in wild type mice is HDL. One of the most frequently employed atherosclerosis models is the Ldlr-/- mouse. On chow diet the mice accumulate of LDL and on a high fat, high cholesterol Western type diet (WTD), VLDL and LDL particles accumulate. As discussed above, PCSK9 overexpression leads to increased levels of LDL-C. Studies with transgenic mice expressing wild type mouse Pcsk9 or human PCSK9 in the liver have shown that increased synthesis of PCSK9 alone is sufficient to increase atherosclerosis in mice when fed a WTD ${ }^{[3,34]}$. However, the lesions were early fatty streak lesions. The transgene is also able to increase atherosclerosis in chow fed Apoe-/- mice but not in WTD fed Ldlr-/- mice. In mice expressing a single copy of the wild type or mutant human PCSK9 genes under control of its own promoter led to more physiological levels of plasma PCSK9 and demonstrated the enhanced ability of the gain of function mutant PCSK9-D374Y to develop atherosclerosis on a high cholesterol diet compared to wild type PCSK9 ${ }^{[22]}$.

The use of the Ldlr-/- mouse as a platform to assess the impact of a variety of other genes in mouse atherogenesis requires costly and time-consuming genetic crosses. This is even more difficult for the exploration of atherosclerosis in other species. A valuable and less onerous approach not requiring genetic crosses involves the injection into appropriate hosts of recombinant hepatic transducing virus expressing PCSK9, especially gain of function mutants. The most frequently employed gain of function human PCSK9 mutant is that at residue 374 in the catalytic domain where aspartic acid is substituted for tyrosine. The endogenous corresponding mouse residue is at position 377. This mutation significantly reduces the autocatalytic cleavage of pro-PCSK $9^{[41]}$. The applicability of this approach is exemplified by Bjørklund et al. ${ }^{[42]}$ who used either human or mouse PCSK9-D374/377Y gain of function mutants in an adenoassociated virus (AAV) substrain 8 vector. Wild type $\mathrm{C} 57 \mathrm{BL} / 6$ mice were injected with various doses of the recombinant virus and fed either the WTD or the Paigen diet which includes sodium cholate. There was a dose dependent increase in plasma lipids, mostly LDL-C, though at the highest dose of virus there was an increase in the VLDL/LDL ratio. Lipids were not as high as is seen in the Ldlr-/- mice fed the same diets, perhaps because the extrahepatic receptor plays a role in the regulation of plasma lipids. The aortic vessels were sampled at 12 weeks of diet feeding. A dose dependent increase in atherosclerotic lesions was observed in the upper vasculature which includes the aortic arch and brachiocephalic artery. The lesions were larger with the feeding of the Paigen diet. Although there was no dose response, the expression of PCSK9 also promoted the formation of advanced lesions with necrotic cores in the aortic root. Hamsters were also injected with the hPCSK9-D374Y AAV virus; they were not as intensively studied but they exhibited hyperlipidemia and early atherosclerotic lesions. The utility of this approach to induce atherosclerosis in a more complex phenotype was illustrated in virus injected diabetic Akita mice, which develop larger lesions than non-diabetic recipients of the virus. It is well known that various mouse strains exhibit differences in susceptibility to atherosclerosis. C57BL/6 mice are the most sensitive, while $129 / \mathrm{Sv}$ and FVB/N strains are more resistant. All 3 strains exhibited comparable hypercholesterolemia on receipt of the human PCSK9D374Y expressing virus, but this did not alter their relative atherosensitivity ${ }^{[43]}$. 
Two studies have compared the AAV8 mediated expression of PCSK9 in male and female mice. The injection of $3 \times 10^{10}$ genome copies of AAV8-PCSK9 into wild type C57BL/6 mice showed a different tropism of the virus with much lower expression in the liver of female mice than male mice ${ }^{[44]}$. This was associated with greater hyperlipidemia in male mice. The gender differences could be equalized by increasing the viral dose administered to female mice by 3 -fold. Another study ${ }^{[45]}$ used an AAV dose of $5 \times$ $10^{11}$ genome copies, which is higher than the highest level employed by Vozenilek et al ${ }^{[44]}$, to express human PCSK9-D374Y in wild type mice. In AAV- hPCSK9 injected mice, males exhibited higher levels of plasma PCSK9 than females but the resultant decrease in hepatic LDLR was higher in females. The authors noted that there was considerable variability in the plasma levels of PCSK9 in both groups. Plasma lipids were measured every 4 weeks after initiation of WTD. Plasma cholesterol increased more slowly with time in male mice expressing hPCSK9 than females and by 20 weeks was only $50 \%$ of that observed in male Ldlr-/mice. Plasma cholesterol levels in female mice expressing hPCSK9, on the other hand, were more similar to female Ldlr-/- mice. The distribution of PCSK9 in the plasma was assessed. In males more than half of the protein was in the HDL fraction while in females almost all of the protein was in the lipid free region. With respect to atherosclerosis assessed by the en face method at the end of the 20 -week diet period, the lesions were $\sim 3$ fold smaller in the male AAV-hPCSK9 treated mice than $L d l r-/$ - mice. In female mice the lesions were similar in size in the $L d l r-/$ - and PCSK9 treated mice. In this study the authors also examined the ability of AAV8 mediated delivery of CRISPR/Cas9 to liver cells to disrupt the Ldlr gene by creating insertion or deletion mutants (INDELs). The efficiency INDEL formation was comparable in male and female mice. However, this too resulted in sex differences in the resulting hyperlipidemia and atherosclerosis. The authors state that with this complexity in virus-based modulation of the LDLR expression it is necessary that "comparisons across sex and strains should be avoided" ${ }^{[45]}$.

AAV-mediated expression of PCSK9 can also be used in atherosclerosis regression models. Wild type C57BL/6 mice were treated with AAV8-mPCSK9-D377Y and fed a WTD for 16 weeks followed by a switch to low fat chow diet with or without an inhibitor of MTP for 6 weeks. Similar to other regression models, the diet switch led to significant decrease in hyperlipidemia which was further enhanced by the MTP inhibitor. While no decrease in lesion size was observed, there was a significant loss in the macrophage content of the aortic root lesions during the regression period accompanied by a drop in blood monocytes ${ }^{[46]}$. Reduced macrophage content is often observed in regressing lesions ${ }^{[47]}$. Hypercholesterolemia promotes monocytosis that contributes to atherogenesis ${ }^{[48]}$.

Although not directly related to PCSK9 function, an alternate approach to the reduction of LDLR levels has employed antisense oligonucleotides to the Ldlr mRNA with a resultant increase in plasma LDL-C levels and the development of lesions in the aortic root, aortic arch and brachiocephalic artery in mice fed a $\mathrm{WTD}^{[49]}$. The macrophage content of these lesions can be substantially reduced by the removal of the antisense oligonucleotides using a sense version of the oligonucleotide.

Endothelial cells, smooth muscle cells, and macrophages in the atherosclerotic lesion also express PCSK9, but at much lower levels ${ }^{[50]}$. Of the three cell types, smooth muscle cells appear to be a major source of PCSK9 in atherosclerotic lesions and in vitro studies have shown that smooth muscle cell-derived PCSK9 can promote LDLR degradation in macrophages in a paracrine fashion ${ }^{[51]}$.

Local effects of PCSK9 are evident from the study of bone marrow chimeras derived by the transfer of bone marrow from hPCSK9tg/Apoe-/- mice into Apoe-/- mice ${ }^{[52]}$. The peritoneal macrophages in the hPCSK9tg/ Apoe-/- mice express both human and murine PCSK9 and have reduced levels of cell surface LDLR and LRP1. Although human PCSK9 is detectable in the plasma of the Apoe-/- recipients of the bone marrow, no 
change in plasma lipids or lesion size was noted. However, hPCSK9 was detected in lesions of the Apoe-/recipients as was an increase in inflammatory Ly $6 \mathrm{C}^{\text {hi }}$ monocytes. A similar study was performed with $\mathrm{Ldlr}-/$ mice but human PCSK9 was not detected in the lesions nor was there an increase in Ly6C ${ }^{\text {hi }}$ cells suggesting that PCSK9 mediated inflammation is LDLR dependent. Upon in vitro stimulation of peritoneal macrophages with LPS, increased levels of proinflammatory Ill $b$ and Tnfa expression was observed in the transgenic macrophages compared to control macrophages. The inflammatory effects of the transgene were also dependent on the presence of the $\operatorname{LDLR}^{[52]}$.

The local influence of PCSK9 has recently been reviewed by Ding et al. ${ }^{[33]}$. From this it is clear that PCSK9 is pro-atherogenic at 2 physiologic levels - by controlling the level of LDL-C as discussed above and by exerting pro-inflammatory influence at the vessel wall level. Disturbed flow, sites at which lesions often develop, activates the production of PCSK9 especially by smooth muscle cells in the presence of dysfunctional endothelial cells. PCSK9 and scavenger receptor LOX-1 expression in macrophages are involved in a positive feedback loop which facilitates the uptake of oxidized LDL, the formation of foam cells and the progression of atherogenesis ${ }^{[54]}$. Upregulation of PCSK9-LOX-1 expression is associated with the activation of reactive oxygen species production that requires the activity of NADPH oxidase.

\section{Atherosclerosis in larger animal models}

The study of murine atherosclerosis in genetically modified mice has provided a wealth of information on the many processes involved in atherogenesis. But this model has limitations. First, mice are small and do not readily furnish the mass of tissue required for detailed biochemical characterization. Second, they do not often develop advanced and complicated lesions that are characteristic of clinically significant atherosclerosis in humans. Third, the distribution of lesions is not exactly similar to those found in humans. For example, obstructive coronary artery lesions are not found in most models of murine atherosclerosis. Finally, the use of in vivo imaging of progressing lesions is not easy in mice. Other larger animal species have been employed in experimental atherosclerosis studies ${ }^{[5,56]}$. Among the models most studied are rabbits (including the Watanabe heredity hypercholesterolemic rabbit with mutant LDLR), nonhuman primates, and pigs.

Pigs are attractive animal models that overcome many of the limitations listed above for mice. A favored subspecies is the miniature Yucatan pig, which is close to the size of human subjects. The advantage of the pig as an experimental model is that pigs have the following similarities with humans: hemodynamics, heart size, cardiac vasculature anatomy, lipid and lipoprotein profile (though they lack apoprotein A-II), and ease of imaging ${ }^{[5]}$. As with mice, there are porcine subspecies that exhibit differential sensitivity to hyperlipidemia and atherosclerosis. The Yucatan pigs and Ossabaw miniature pigs are relatively sensitive strains among the studied subspecies, while the Landrace pigs are relatively resistant. This relative atherosensitivity is not altered by the overexpression of PCSK9. Transgenic Yucatan pigs expressing the human gain of function mutant PCSK9-D374Y in the liver were hyperlipidemic with increased plasma LDL levels on both low fat and high fat, high cholesterol (HFHC) diet and develop atherosclerosis on the HFHC diet $^{[58]}$. Similar results were obtained by liver expression of the chimpanzee PCSK9-D374Y gain of function mutant in Ossabaw miniature pigs but not Landrace pigs ${ }^{[59]}$. Atherosclerosis is found in ascending and thoracic aorta, coronary arteries (i.e., left anterior descending and right coronary artery), abdominal aorta, and iliofemoral arteries. This is accentuated by the feeding of the atherogenic diet. Femoral artery disease may also be found especially with feeding the atherogenic diet $^{[60]}$. 


\section{CONCLUSION}

The understanding of the role of PCSK9 in the regulation of the LDLR, lipid homeostasis, and inflammation has led to important therapeutic possibilities. As pointed out above, the loss of function of PCSK9 leads to an increase in the function of the LDLR and hypocholesterolemia, indicating that the protein does function under steady state. This can be induced by PCSK9 monoclonal antibody or antisense oligonucleotides treatment. Indeed, germline loss of function leads to lifetime hypocholesterolemia with very low coronary artery disease risk ${ }^{[1]}$.

\section{DECLARATIONS}

\section{Authors' contributions}

Made substantial contributions to conception, research and writing of the review: Getz GS, Reardon CA

\section{Availability of data and materials}

Not applicable.

\section{Financial support and sponsorship}

None.

\section{Conflicts of interest}

Both authors declared that there are no conflicts of interest.

\section{Ethical approval and consent to participate}

Not applicable.

\section{Consent for publication}

Not applicable.

\section{Copyright}

(c) The Author(s) 2021.

\section{REFERENCES}

1. Goldstein JL, Brown MS. A century of cholesterol and coronaries: from plaques to genes to statins. Cell 2015;161:161-72. DOI PubMed PMC

2. Zhang L, Reue K, Fong LG, Young SG, Tontonoz P. Feedback regulation of cholesterol uptake by the LXR-IDOL-LDLR axis. Arterioscler Thromb Vasc Biol 2012;32:2541-6. DOI PubMed PMC

3. Abifadel M, Varret M, Rabès JP, et al. Mutations in PCSK9 cause autosomal dominant hypercholesterolemia. Nat Genet 2003;34:1546. DOI PubMed

4. Timms KM, Wagner S, Samuels ME, et al. A mutation in PCSK9 causing autosomal-dominant hypercholesterolemia in a Utah pedigree. Hum Genet 2004;114:349-53. DOI PubMed

5. Leren TP. Mutations in the PCSK9 gene in Norwegian subjects with autosomal dominant hypercholesterolemia. Clin Genet 2004;65:419-22. DOI PubMed

6. Schlüter K-D, Wolf A, Schreckenberg R. Coming back to physiology: Extra hepatic functions of proprotein convertase Subtilisin/Kexin type 9. Front Physiol 2020;11:598649. DOI PubMed PMC

7. Stoekenbroek RM, Lambert G, Cariou B, Hovingh GK. Inhibiting PCSK9 - biology beyond LDL control. Nat Rev Endocrinol 2019;15:52-62. DOI PubMed

8. Schulz R, Schlüter KD, Laufs U. Molecular and cellular function of the proprotein convertase subtilisin/kexin type 9 (PCSK9). Basic Res Cardiol 2015;110:4. DOI PubMed PMC

9. Lambert G, Sjouke B, Choque B, Kastelein JJ, Hovingh GK. The PCSK9 decade. J Lipid Res 2012;53:2515-24. DOI PubMed PMC

10. Gustafsen C, Kjolby M, Nyegaard M, et al. The hypercholesterolemia-risk gene SORT1 facilitates PCSK9 secretion. Cell Metab 2014;19:310-8. DOI PubMed

11. Tavori H, Christian D, Minnier J, et al. PCSK9 association with lipoprotein(a). Circ Res 2016;119:29-35. DOI PubMed PMC

12. Grefhorst A, McNutt MC, Lagace TA, Horton JD. Plasma PCSK9 preferentially reduces liver LDL receptors in mice. J Lipid Res 2008;49:1303-11. DOI PubMed PMC

13. Ni YG, Condra JH, Orsatti L, et al. A proprotein convertase subtilisin-like/kexin type 9 (PCSK9) C-terminal domain antibody antigen- 
binding fragment inhibits PCSK9 internalization and restores low density lipoprotein uptake. J Biol Chem 2010;285:12882-91. DOI PubMed PMC

14. Borchardt RA, Davis RA. Intrahepatic assembly of very low density lipoproteins. Rate of transport out of the endoplasmic reticulum determines rate of secretion. J Biol Chem 1987;262:16394-402. PubMed

15. Blasiole DA, Davis RA, Attie AD. The physiological and molecular regulation of lipoprotein assembly and secretion. Mol Biosyst 2007;3:608-19. DOI PubMed

16. Shapiro MD, Fazio S. PCSK9 and atherosclerosis - lipids and beyond. J Atheroscler Thromb 2017;24:462-72. DOI PubMed PMC

17. Twisk J, Gillian-Daniel DL, Tebon A, Wang L, Barrett PH, Attie AD. The role of the LDL receptor in apolipoprotein B secretion. $J$ Clin Invest 2000;105:521-32. DOI PubMed PMC

18. Horton JD, Cohen JC, Hobbs HH. Molecular biology of PCSK9: its role in LDL metabolism. Trends Biochem Sci 2007;32:71-7. DOI PubMed PMC

19. Davignon J, Dubuc G, Seidah NG. The influence of PCSK9 polymorphisms on serum low-density lipoprotein cholesterol and risk of atherosclerosis. Curr Atheroscler Rep 2010;12:308-15. DOI PubMed

20. Rosenson RS, Hegele RA, Fazio S, Cannon CP. The evolving future of PCSK9 inhibitors. J Am Coll Cardiol 2018;72:314-29. DOI PubMed

21. Sabatine MS. PCSK9 inhibitors: clinical evidence and implementation. Nat Rev Cardiol 2019;16:155-65. DOI PubMed

22. Raal FJ, Giugliano RP, Sabatine MS, et al. PCSK9 inhibition-mediated reduction in Lp(a) with evolocumab: an analysis of 10 clinical trials and the LDL receptor's role. J Lipid Res 2016;57:1086-96. DOI PubMed PMC

23. Ray KK, Wright RS, Kallend D, et al. Two phase 3 trials of inclisiran in patients with elevated LDL cholesterol. N Engl J Med 2020;382:1507-19. DOI PubMed

24. Jeong HJ, Lee HS, Kim KS, Kim YK, Yoon D, Park SW. Sterol-dependent regulation of proprotein convertase subtilisin/kexin type 9 expression by sterol-regulatory element binding protein-2. J Lipid Res 2008;49:399-409. DOI PubMed

25. Sun L, Yang X, Li Q, et al. Activation of adiponectin receptor regulates proprotein convertase Subtilisin/Kexin Type 9 expression and inhibits lesions in ApoE-deficient mice. Arterioscler Thromb Vasc Biol 2017;37:1290-300. DOI PubMed

26. Rashid S, Curtis DE, Garuti R, et al. Decreased plasma cholesterol and hypersensitivity to statins in mice lacking Pcsk9. Proc Natl Acad Sci U S A 2005;102:5374-9. DOI PubMed PMC

27. Zaid A, Roubtsova A, Essalmani R, et al. Proprotein convertase subtilisin/kexin type 9 (PCSK9): hepatocyte-specific low-density lipoprotein receptor degradation and critical role in mouse liver regeneration. Hepatology 2008;48:646-54. DOI PubMed

28. Lagace TA, Curtis DE, Garuti R, et al. Secreted PCSK9 decreases the number of LDL receptors in hepatocytes and in livers of parabiotic mice. J Clin Invest 2006;116:2995-3005. DOI PubMed PMC

29. Herbert B, Patel D, Waddington SN, et al. Increased secretion of lipoproteins in transgenic mice expressing human D374Y PCSK9 under physiological genetic control. Arterioscler Thromb Vasc Biol 2010;30:1333-9. DOI PubMed

30. Maxwell KN, Breslow JL. Adenoviral-mediated expression of Pcsk9 in mice results in a low-density lipoprotein receptor knockout phenotype. Proc Natl Acad Sci U S A 2004;101:7100-5. DOI PubMed PMC

31. Park SW, Moon YA, Horton JD. Post-transcriptional regulation of low density lipoprotein receptor protein by proprotein convertase subtilisin/kexin type 9a in mouse liver. J Biol Chem 2004;279:50630-8. DOI PubMed

32. Lalanne F, Lambert G, Amar MJ, et al. Wild-type PCSK9 inhibits LDL clearance but does not affect apoB-containing lipoprotein production in mouse and cultured cells. J Lipid Res 2005;46:1312-9. DOI PubMed

33. Denis M, Marcinkiewicz J, Zaid A, et al. Gene inactivation of proprotein convertase subtilisin/kexin type 9 reduces atherosclerosis in mice. Circulation 2012;125:894-901. DOI PubMed

34. Tavori H, Giunzioni I, Predazzi IM, et al. Human PCSK9 promotes hepatic lipogenesis and atherosclerosis development via apoE- and LDLR-mediated mechanisms. Cardiovasc Res 2016;110:268-78. DOI PubMed PMC

35. Seidah NG, Benjannet S, Wickham L, et al. The secretory proprotein convertase neural apoptosis-regulated convertase 1 (NARC-1): liver regeneration and neuronal differentiation. Proc Natl Acad Sci U S A 2003;100:928-33. DOI PubMed PMC

36. Rashid S, Tavori H, Brown PE, et al. Proprotein convertase subtilisin kexin type 9 promotes intestinal overproduction of triglyceriderich apolipoprotein B lipoproteins through both low-density lipoprotein receptor-dependent and -independent mechanisms. Circulation 2014;130:431-41. DOI PubMed PMC

37. Levy E, Ben Djoudi Ouadda A, et al. PCSK9 plays a significant role in cholesterol homeostasis and lipid transport in intestinal epithelial cells. Atherosclerosis 2013;227:297-306. DOI PubMed

38. Wang Y, Huang Y, Hobbs HH, Cohen JC. Molecular characterization of proprotein convertase subtilisin/kexin type 9-mediated degradation of the LDLR. J Lipid Res 2012;53:1932-43. DOI PubMed PMC

39. Thedrez A, Sjouke B, Passard M, et al. Proprotein Convertase Subtilisin Kexin Type 9 Inhibition for Autosomal Recessive Hypercholesterolemia-Brief Report. Arterioscler Thromb Vasc Biol 2016;36:1647-50. DOI PubMed

40. Choi S, Aljakna A, Srivastava U, et al. Decreased APOE-containing HDL subfractions and cholesterol efflux capacity of serum in mice lacking Pcsk9. Lipids Health Dis 2013;12:112. DOI PubMed PMC

41. Benjannet S, Rhainds D, Essalmani R, et al. NARC-1/PCSK9 and its natural mutants: zymogen cleavage and effects on the low density lipoprotein (LDL) receptor and LDL cholesterol. J Biol Chem 2004;279:48865-75. DOI PubMed

42. Bjørklund MM, Hollensen AK, Hagensen MK, et al. Induction of atherosclerosis in mice and hamsters without germline genetic engineering. Circ Res 2014;114:1684-9. DOI PubMed

43. Roche-Molina M, Sanz-Rosa D, Cruz FM, et al. Induction of sustained hypercholesterolemia by single adeno-associated virusmediated gene transfer of mutant hPCSK9. Arterioscler Thromb Vasc Biol 2015;35:50-9. DOI PubMed 
44. Vozenilek AE, Blackburn CMR, Schilke RM, et al. AAV8-mediated overexpression of mPCSK9 in liver differs between male and female mice. Atherosclerosis 2018;278:66-72. DOI PubMed PMC

45. Jarrett KE, Lee C, De Giorgi M, et al. Somatic editing of ldlr with adeno-associated viral-CRISPR is an efficient tool for atherosclerosis research. Arterioscler Thromb Vasc Biol 2018;38:1997-2006. DOI PubMed PMC

46. Peled M, Nishi H, Weinstock A, et al. A wild-type mouse-based model for the regression of inflammation in atherosclerosis. PLoS One 2017;12:e0173975. DOI PubMed PMC

47. Fisher EA. Regression of atherosclerosis: the journey from the liver to the plaque and back. Arterioscler Thromb Vasc Biol 2016;36:226-35. DOI PubMed PMC

48. Swirski FK, Libby P, Aikawa E, et al. Ly-6Chi monocytes dominate hypercholesterolemia-associated monocytosis and give rise to macrophages in atheromata. J Clin Invest 2007;117:195-205. DOI PubMed PMC

49. Basu D, Hu Y, Huggins LA, et al. Novel reversible model of atherosclerosis and regression using oligonucleotide regulation of the LDL receptor. Circ Res 2018;122:560-7. DOI PubMed PMC

50. Ding Z, Liu S, Wang X, et al. Hemodynamic shear stress via ROS modulates PCSK9 expression in human vascular endothelial and smooth muscle cells and along the mouse aorta. Antioxid Redox Signal 2015;22:760-71. DOI PubMed PMC

51. Ferri N, Tibolla G, Pirillo A, et al. Proprotein convertase subtilisin kexin type 9 (PCSK9) secreted by cultured smooth muscle cells reduces macrophages LDLR levels. Atherosclerosis 2012;220:381-6. DOI PubMed

52. Giunzioni I, Tavori H, Covarrubias R, et al. Local effects of human PCSK9 on the atherosclerotic lesion. J Pathol 2016;238:52-62. DOI PubMed PMC

53. Ding Z, Pothineni NVK, Goel A, Lüscher TF, Mehta JL. PCSK9 and inflammation: role of shear stress, pro-inflammatory cytokines, and LOX-1. Cardiovasc Res 2020;116:908-15. DOI PubMed

54. Ding Z, Liu S, Wang X, et al. PCSK9 regulates expression of scavenger receptors and ox-LDL uptake in macrophages. Cardiovasc Res 2018;114:1145-53. DOI PubMed

55. Getz GS, Reardon CA. Animal models of atherosclerosis. Arterioscler Thromb Vasc Biol 2012;32:1104-15. DOI PubMed PMC

56. Shim J, Al-Mashhadi RH, Sørensen CB, Bentzon JF. Large animal models of atherosclerosis--new tools for persistent problems in cardiovascular medicine. J Pathol 2016;238:257-66. DOI PubMed

57. Lee YT, Laxton V, Lin HY, et al. Animal models of atherosclerosis. Biomed Rep 2017;6:259-66. DOI PubMed PMC

58. Al-Mashhadi RH, Sørensen CB, Kragh PM, et al. Familial hypercholesterolemia and atherosclerosis in cloned minipigs created by DNA transposition of a human PCSK9 gain-of-function mutant. Sci Transl Med 2013;5:166ra1. DOI PubMed

59. Yuan F, Guo L, Park KH, et al. Ossabaw pigs with a PCSK9 gain-of-function mutation develop accelerated coronary atherosclerotic lesions: A novel model for preclinical studies. $J$ Am Heart Assoc 2018;7:e06207. DOI PubMed PMC

60. Hedayat AF, Park KH, Kwon TG, et al. Peripheral vascular atherosclerosis in a novel PCSK9 gain-of-function mutant Ossabaw miniature pig model. Transl Res 2018;192:30-45. DOI PubMed PMC 